\title{
Fusion arrest and collapse phenomena due to Kerr-nonlinearity in quadratic media
}

\author{
Johansen, Steffen Kjær; Bang, Ole; Sørensen, Mads Peter
}

Published in:

Nonlinear Optics: Materials, Fundamentals, and Applications, 2000. Technical Digest

Link to article, DOI:

10.1109/NLO.2000.883589

Publication date:

2000

Document Version

Publisher's PDF, also known as Version of record

Link back to DTU Orbit

Citation (APA):

Johansen, S. K., Bang, O., \& Sørensen, M. P. (2000). Fusion arrest and collapse phenomena due to Kerrnonlinearity in quadratic media. In Nonlinear Optics: Materials, Fundamentals, and Applications, 2000. Technical Digest (pp. 99-101). Optical Society of America. https://doi.org/10.1109/NLO.2000.883589

\section{General rights}

Copyright and moral rights for the publications made accessible in the public portal are retained by the authors and/or other copyright owners and it is a condition of accessing publications that users recognise and abide by the legal requirements associated with these rights.

- Users may download and print one copy of any publication from the public portal for the purpose of private study or research.

- You may not further distribute the material or use it for any profit-making activity or commercial gain

- You may freely distribute the URL identifying the publication in the public portal 


\title{
Fusion arrest and collapse phenomena due to Kerr-nonlinearity in quadratic media
}

\author{
Steffen Kjær Johansen, Ole Bang and Mads Peter Sørensen \\ Department of Mathematical Modelling, Technical University of Denmark, \\ DK-2800 Lyngby, Denmark \\ Tel.: (+45) 452531 0.9; Fax: (+45) 459312 35; E-mail: skj@imm.dtu.dk
}

\begin{abstract}
Emphasizing collapse phenomena it is investigated to what extend the always present cubic nonlinearity affects the properties of soliton interaction in quadratic bulk media. An effective particle approach is applied and verified by numerical simulations.

(C) 2000 Optical Society of America

OCIS codes: (190.0190) Nonlinear optics; (260.0260) Physical optics
\end{abstract}

The understanding of how and to what extend the cubic nonlinearity affects beam propagation and spatial soliton formation in quadratic media is of vital importance not only in fundamental nonlinear physics but also in the applied field of rewritable optical circuitry, i.e. switching devices, junctions etc. [1, 2].

All quadratic materials have an inherent cubic nonlinearity that becomes important at high intensities or when the fundamental wave (FW) and its second harmonic (SH) do not meet the phase-matching condition. Recently also very strong induced cubic nonlinearities have been achieved via quasi-phase-matching techniques[3, 4].
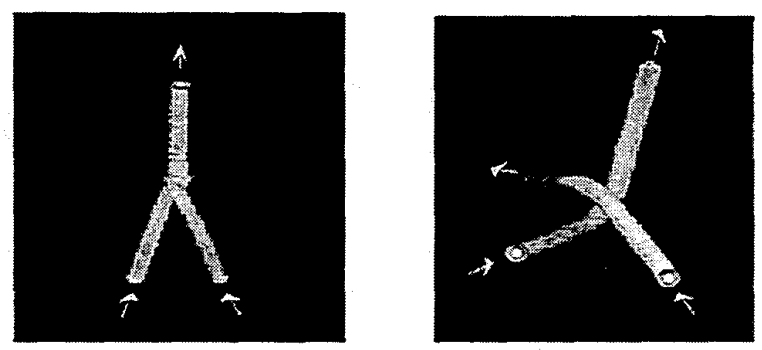

Fig. 1. Fusion and spiraling. Examples of spatial soliton interactions in bulk media[5].

We consider beam propagation under type-I SHG conditions in lossless bulk $\chi^{(2)}$ materials with a nonvanishing $\chi^{(3)}$ nonlinearity. It is known that in pure $\chi^{(2)}$ systems a single soliton can never collapse[6] whereas in systems with both nonlinearities stable single soliton propagation can only be achieved for small effective $\chi^{(3)}$ values and low powers[7]. The well-known system of normalized nonlinear equations describing the propagation of the slowly varying envelopes are[8]

$$
\begin{aligned}
& i \frac{d A_{1}}{d z}+\frac{1}{2} \nabla_{\perp}^{2} A_{1}+A_{2} A_{1}^{*}+\gamma\left(\frac{1}{2}\left|A_{1}\right|^{2}+\left|A_{2}\right|^{2}\right) A_{1}=0 \\
& i \frac{d A_{2}}{d z}+\frac{1}{4} \nabla_{\perp}^{2} A_{2}-\beta A_{2}+A_{1}^{2}+\gamma\left(\left|A_{2}\right|^{2}+2\left|A_{1}\right|^{2}\right) A_{2}=0
\end{aligned}
$$

where $\beta$ is the effective phase-mismatch parameter and $\gamma$ determines the relative strength of the effective $\chi^{(3)}$ nonlinearity to that of the effective $\chi^{(2)}$ nonlinearity. $A_{1}$ and $A_{2}$ are, respectively, the FW and the SH. The system can be derived from a Lagrangian density. In the case of two interacting solitons

$$
A_{1}=A_{1}^{(1)}+A_{1}^{(2)}, \quad A_{2}=A_{2}^{(1)}+A_{2}^{(2)}
$$


the extra terms in the Lagrangian density, containing contributions from both solitons, can be treated as first order perturbations. The solitons are each characterized by three parameters ( $\lambda$ defining the soliton profile and $\nu_{x}$ and $\nu_{y}$ accounting for transverse velocities) which are then allowed slow adiabatic variation. The first order correction to the Lagrangian describes how the soliton parameters change during propagation. Through the effective particle approach the dimensionality of the system can be reduced yielding the following first order correction to the Lagrangian in case of initially identical solitons

$$
L=\frac{1}{2} M_{R} \dot{R}^{2}-\frac{1}{2} M_{\phi} \dot{\phi}^{2}-U_{e f f}
$$

where

$$
M_{R}=\pi \int_{0}^{\infty}\left[\left|V_{s}\right|^{2}+2\left|W_{s}\right|^{2}\right] r \mathrm{~d} r, \quad M_{\phi}=2 \frac{\partial M_{R}}{\partial \lambda}
$$

$R$ and $\phi$ are, respectively, the relative distance between soliton centers and the relative phase between the solitons. The effective potential $U_{\text {eff }}$ consists of three terms: the classical centrifugal potential barrier and an interaction integral for each of the nonlinearities consisting of various coupling terms between the two solitons.
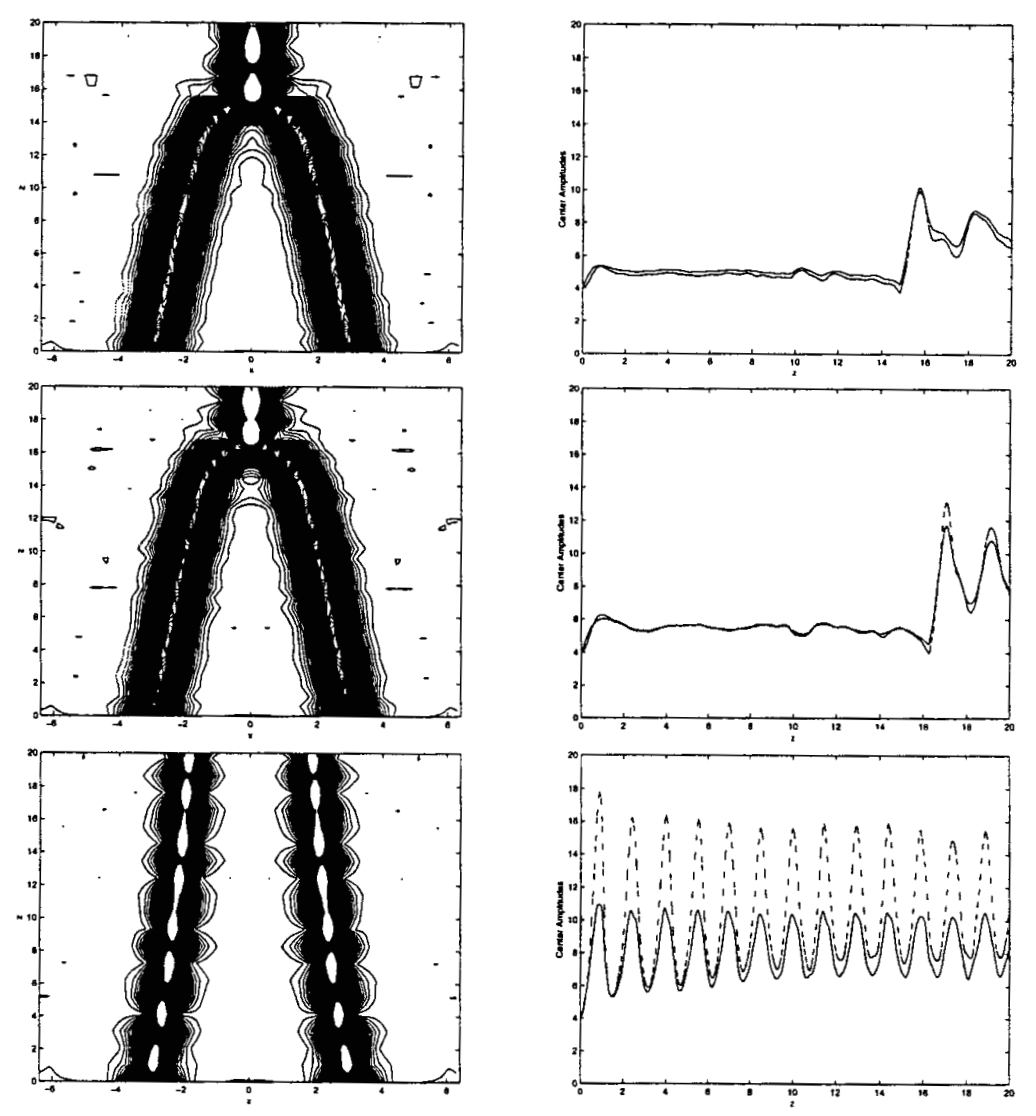

Fig. 2. Fusion arrest due to $\chi^{(3)}$ nonlinearity. In the contour plot only the FW's are shown. Top: $\gamma=0$, Middle: $\gamma=0.01$, Bottom: $\gamma=0.05$. 
The model is based on the theory formulated in [5] for the pure $\chi^{(2)}$ case. The introduction of a $\chi^{(3)}$ nonlinearity gives rise to qualitatively different behavior for the different scenarios, i.e. fusion, repulsion and spiraling. As an example we mention the case of fusion where we have observed that the introduction of the $\chi^{(3)}$ nonlinearity leads to an arrest of the fusion process. This is shown in figure 2 where to identical solitons with no relative phase difference collide.

The collapse effect introduced by the $\chi^{(3)}$ nonlinearity may lead to other qualitatively new behaviors of the system. The system is investigated analytically under a Gaussian approximation in the effective particle approach and the results are verified numerically.

\section{References}

1. M. Klotz, H. Meng, G. J. Salamo, M. Segev, S. R. Montgomery, "Fixing the photorefractive soliton," Optics Letters 24 $77-79$ (1999).

2. M. Klotz, M. Crosser, G. J. Salamo and M. Segev, "Fixing solitonic waveguides in photorefractive strontium barium niobate", in Nonlinear Guided Waves and Their Applications, OSA Technical Digest (Optical Society of America, Washington, D.C., 1998), pp. 417-419.

3. C. B. Clausen, O. Bang and Y. S. Kivshar, "Spatial solitons and induced Kerr effects in QPM media," Phys. Rev. Lett. 78, 4749-4752 (1997).

4. O. Bang, C. B. Clausen, P. L. Christiansen and L. Torner, "Engineering competing nonlinearities," Optics Letters 24 $1413-1415$ (1999).

5. A. V. Buryak and V. V. Steblina, "Soliton collisions in bulk quadratic media: comprehensive analytical and numerical study," J. Opt. Soc. Am. B 16, 245-255 (1999).

6. L. Bergé, O. Bang, J. J. Rasmussen and V. K. Mezentsev, "Self-focusing and solitonlike structures in materials with competing quadratic and cubic nonlinearities," Phys. Rev. E 55, 3555-3570 (1997).

7. O. Bang, Y.S. Kivshar, A. V. Buryak, A. De Rossi and S. Trillo, "Two-dimensional solitary waves in media with quadratic and cubic nonlinearity," Phys. Rev. E 58, 5057-5069 (1998)

8. O. Bang, "Dynamical equations for wave packets in materials with both quadratic and cubic responses," J. Opt. Soc. Am. B 14, 51-61 (1997) 